\title{
Noise calibration, delay coherence and SNR walls for signal detection
}

\author{
Rahul Tandra \\ Department of Electrical Engineering and \\ Computer Sciences \\ University of California, Berkeley \\ Email: tandra@eecs.berkeley.edu
}

\author{
Anant Sahai \\ Department of Electrical Engineering and \\ Computer Sciences \\ University of California, Berkeley \\ Email: sahai@eecs.berkeley.edu
}

\begin{abstract}
In this paper we consider the problem of robustly detecting the presence/absence of signals in uncertain low SNR environments. Our previous results have shown the existence of fundamental SNR thresholds called SNR walls below which robust detection is impossible. For signals with narrowband pilots, we had shown earlier that a simple modification to the matched filter provides significant robustness gains. This technique is called runtime noise calibration. In this paper we show that runtime noise calibration can also be applied to improve the robustness of other feature detectors. We use a $50 \%$ dutycycle pulse-amplitude-modulated signal as an example to illustrate the gains from noise calibration.

Robustness results for this example give us important insights which also apply in the general case of cyclostationary signals. Our results suggests that for cyclostationary signals, frequency-selective fading and uncertain noise color are the main reasons for detector non-robustness. Furthermore, we introduce the notion of delay coherence for frequency-selective channels. We also characterize the location of the SNR wall as a function of the delaycoherence time of the channel.
\end{abstract}

\section{INTRODUCTION}

In this paper we consider the problem of detecting the presence/absence of weak signals in noisy uncertain environments. This problem is important in many practical situations. Our main motivation to consider this problem stems from secondary users trying to opportunistically reuse primary spectrum [1]. Such opportunistic secondary systems are allowed to recycle underutilized primary spectrum as long as they do not cause interference to any primary user. In order to guarantee noninterference, it is known that secondary users must be able to sense weak primary signals [2], [3].

In low SNR environments small modeling uncertainties are unavoidable and robustness to them has been shown to be a fundamental performance metric.
Both theoretical and experimental results have shown the existence of the phenomenon of SNR walls fundamental SNR thresholds below which robustness to uncertainties is impossible [4]-[7]. In particular, all commonly used detection strategies like the radiometer (energy detector) and the matched filter suffer from SNR wall limitations [4], [8]. For a better understanding of the SNR wall phenomenon, we give an alternate samplecomplexity based interpretation in Section II.

Here our goal is to explore signal processing techniques that improve detector robustness. One such technique was introduced in [4] for the case of matched filters trying to detect narrowband pilots. The key idea was to use in-band measurements at frequencies where the pilot is absent to calibrate the noise statistics at the pilot frequencies. This technique was called runtime noise calibration. It exploits the localized nature of the signal feature (pilot tone) within the whole band of interest. This raises the following obvious question: can we obtain similar noise-calibration gains for other classes of signals?

In many communications schemes the statistics of the transmitted signals are periodic. This is due to inherent periodicities in the system like the modulation rate, carrier frequency, etc. It has been argued that such signals can be modeled to be widesense cyclostationary [9][11]. Feature detectors are a class of detectors which can be used to distinguish cyclostationary signals from stationary noise. They were first proposed by William Gardner [12], [13]. These detectors were believed to be attractive candidates to solve the problem of robustly detecting very weak signals in noise [14].

Feature detection can be thought as a two step process: A transformation from the time-domain into the feature domain; followed by a hypothesis test in this new domain. One particular detection algorithm is to focus on 
a single signal feature in the transform domain, and test its presence/absence to decide whether the primary signal is present or absent. This is called a single-cycle feature detector [12]. The robustness analysis of the single-cycle feature detector was given in [15]. It was shown that in a block fading model the single-cycle feature detector also suffers from SNR wall limitations, and the SNR wall decays as $\frac{1}{\sqrt{N_{c}}}$, where $N_{c}$ is the coherence time of the channel.

However, in [15], the key insights were obscured by the notational complexity of feature detection. Also, the SNR wall in [15] is derived as a function of the channel-coherence time, without giving any insight into the physical nature of the channel-coherence time. More importantly, these results do not provide any intuition into whether run-time noise calibration is feasible for feature detectors. To answer these questions we need to move to a simpler model.

So, we focus on pulse-amplitude-modulated (PAM) signals, which are an important class of cyclostationary signals. Curiously, signals modulated with Sinc pulses do not exhibit cyclostationary features. One explanation for this is that under the Sinc modulation, the signal is effectively stationary. An alternative explanation is that the Sinc pulse has 100\% spectral efficiency. A signal using only half the available bandwidth is a trivial example of a signal with spectral redundancy. Despite being a stationary signal, noise calibration gains for this example were shown in [4]. This suggests that noise calibration gains might also be feasible for cyclostationary signals that seemingly have no spectral redundancy.

The simplest example of a cyclostationary signal with seemingly no spectral redundancy is a $50 \%$-duty-cycle PAM signal. The analysis of this example using feature detectors is notationally complex. Instead, we analyze this example using a simple energy-detection based detection algorithm. This algorithm is conceptually similar to the single-cycle feature detection algorithm considered in [15]. We derive the SNR wall results for this detector and show that its robustness performance is comparable to the robustness of the single-cycle feature detector, i.e., the SNR walls for both detectors scale as $\frac{1}{\sqrt{N_{c}}}$.

In this example, the coherence time corresponds to the smallest time over which the impulse response of the channel changes significantly. Since the simplest example of a change in impulse response corresponds to a change in the delay for a pure-delay fading process, we call this the delay-coherence time of the channel and denote it by $D_{c}$. It is shown in Section IV that the delaycoherence time can be significantly different than the tra- ditional phase-coherence and magnitude-coherence times of the channel.

More importantly, we show that a simple modification to this detector will gives run-time noise calibration gains. An analytic expression for the SNR wall for the modified detector is derived, and this shows that the SNR wall for the 50\%-PAM signal (with uncertain noise color) scales as $\frac{1}{\sqrt{D_{c}}}$ with the delay-coherence time of the channel. This scaling is different than the $O\left(\frac{1}{N_{c}}\right)$ scaling of the SNR wall for the matched filter, where $N_{c}$ is the phase-coherence time of the channel.

Table I gives the analogies between noise-calibration gains in the frequency domain (matched filter) and the noise-calibration gains in the time-domain (50\%-dutycycle PAM signal).

\section{ROBUST SENSING PROBLEM SETUP AND BACKGROUND REVIEW}

In this section we briefly recap the robustness results from [4]. The readers are encouraged to read the paper for complete details. In discrete time, the sensing problem can be formulated as a binary hypothesis test with the following hypotheses:

$$
\begin{aligned}
& \mathcal{H}_{0}: Y[n]=W[n] \\
& \mathcal{H}_{1}: Y[n]=H(X)[n]+W[n],
\end{aligned}
$$

for $n=1,2, \cdots, N$. Here $X[n]$ are the samples of the signal of interest, $W[n]$ are samples of noise, $Y[n]$ are the received signal samples and $H($.$) is a linear$ time-varying fading operator. The signal is assumed to be independent of the noise and fading process. The noise process $\{W[n]\}_{n=1, \cdots}$ is modeled to have any distribution $\mathcal{W}$ from a set of possible distributions $\widehat{\mathbb{W}}_{\rho, \lambda}$, $\rho>\lambda \geq 1$. This set is called the noise uncertainty set. The exact mathematical model for the distributions in $\widehat{\mathbb{W}}_{\rho, \lambda}$ is given in [4], and we use this model in the rest of the paper.

The parameter $\rho$ in $\widehat{\mathbb{W}}_{\rho, \lambda}$ is used to quantify the amount of uncertainty in the noise power and the parameter $\lambda$ is used to quantify the uncertainty in the color of the noise process. For the special case when $\lambda=1$, the noise uncertainty model reduces to a 'white' noise uncertainty model. For notational convenience denote the 'white' noise uncertainty set by $\mathbb{W}_{\rho}$. Figure 1 describes the possible power spectral densities under the 'white' and colored noise uncertainty models pictorially.

Under the noise uncertainty model given above, it was shown that the radiometer and the matched filter have fundamental SNR thresholds, called SNR walls, below 


\begin{tabular}{|c|c|c|c|}
\hline Signal model & Strategy & Key reason for non-robustness & SNR wall scaling \\
\hline $\begin{array}{c}\text { Unknown or 'white' signal } \\
\text { with a power constraint }\end{array}$ & Energy detection & Uncertain noise power & \multirow{2}{*}{$O(1)$} \\
\cline { 2 - 3 } 'White' signal with & Optimal detection & Uncertain noise moments & \\
\cline { 2 - 3 } narrowband pilot & $\begin{array}{c}\text { Matched filter with } \\
\text { run-time noise calibration }\end{array}$ & $\begin{array}{c}\text { Finite phase-coherence time } \\
\text { and uncertain noise power } \\
\text { and uncertain noise color }\end{array}$ & \multirow{2}{*}{$O\left(\frac{1}{N_{c}}\right)$} \\
\hline $\begin{array}{c}50 \% \text {-duty-cycle pulse } \\
\text { amplitude modulated signal } \\
\text { (example of a } \\
\text { cyclostationary signal) }\end{array}$ & Detector defined in (12) & $\begin{array}{c}\text { Finite delay-coherence time } \\
\text { and uncertain noise power }\end{array}$ & \multirow{2}{*}{$O\left(\frac{1}{\sqrt{D_{c}}}\right)$} \\
\cline { 2 - 3 } & $\begin{array}{c}\text { Detector defined in (16) } \\
\text { (run-time noise calibration) }\end{array}$ & $\begin{array}{c}\text { Finite delay-coherence time } \\
\text { and uncertain noise color }\end{array}$ & \\
\hline
\end{tabular}

TABLE I

COMPARISON OF ROBUSTNESS RESULTS FOR DIFFERENT CLASSES OF SIGNAL MODELS.

which robust detection ${ }^{1}$ is impossible [4]. The SNR wall expressions for the radiometer and the matched filter are

$$
S N R_{\text {wall }}^{\text {energy }}=\frac{\rho^{2}-1}{\rho},
$$

and

$$
S N R_{\text {wall }}^{m f}=\frac{1}{N_{c} \cdot \theta}\left(\frac{\rho^{2}-1}{\rho}\right),
$$

where $\theta$ is the fraction of the total signal power allocated to the known pilot tone, and $N_{c}$ is the phase-coherence time of the channel.

We now give an alternate interpretation for SNR walls using the notion of sample complexity. Consider the robust detection problem in (1). Assume that the signal and noise samples are independent and identically distributed (iid), and the noise distribution lies in the white noise uncertainty set $\mathbb{W}_{\rho}$. Let the detector teststatistic be given by

$$
T(\mathbf{Y}):=\frac{1}{N} \sum_{n=1}^{N} \phi(Y[n]) \underset{\mathcal{H}_{0}}{\stackrel{\mathcal{H}_{1}}{\gtrless}} \gamma,
$$

where $\phi(\cdot)$ is a known deterministic function and $\gamma$ is the detector threshold. Denote the SNR wall for this detector by $S N R_{\text {wall }}^{T}$. Let $S N R>S N R_{\text {wall }}^{T}$. Then, by the definition of an SNR wall (see [4] for a formal definition) we know that any $P_{F A}<\frac{1}{2}$ and $P_{M D}<\frac{1}{2}$ can be robustly achieved. That is, we can choose a detection threshold $\gamma$ such that

$$
\begin{aligned}
P_{F A} & =\max _{\mathcal{W} \in \mathbb{W}_{\rho}} \mathcal{P}_{\mathcal{W}}\left(T(\mathbf{Y})>\gamma \mid \mathcal{H}_{0}\right), \\
P_{M D} & =\max _{\mathcal{W} \in \mathbb{W}_{\rho}} \mathcal{P}_{\mathcal{W}}\left(T(\mathbf{Y})<\gamma \mid \mathcal{H}_{1}\right)
\end{aligned}
$$

${ }^{1}$ See [4] for a formal definition of robust detection.
Eliminating $\gamma$ from (5) we can solve for $N$ as a function of the SNR, $P_{F A}, P_{M D}$ and $\rho$. Hence, we can write

$$
N=\mu\left(S N R, P_{F A}, P_{M D}, \rho\right) .
$$

This is called the sample complexity of the detector. For any reasonable detector the sample complexity increases as the SNR decreases, i.e., $\mu\left(S N R, P_{F A}, P_{M D}, \rho\right)$ is a monotonically decreasing function of SNR. Under this monotonicity assumption, it turns out that

$$
\lim _{S N R \downarrow S N R_{\text {wall }}^{T}} \mu\left(S N R, P_{F A}, P_{M D}, \rho\right)=\infty .
$$

Equation (7) gives an alternate interpretation for an SNR wall. It tells us that the SNR wall for a detector is the SNR threshold at which the sample complexity approaches $\infty$.

From [4] the sample complexity of the radiometer is

$$
N \approx \frac{2\left[\mathcal{Q}^{-1}\left(P_{F A}\right)-\mathcal{Q}^{-1}\left(1-P_{M D}\right)\right]^{2}}{\left[S N R-\left(\frac{\rho^{2}-1}{\rho}\right)\right]^{2}},
$$

where $\mathcal{Q}^{-1}(\cdot)$ is the inverse of the Gaussian tail probability function. Again from [4], the sample complexity for the matched filter is

$$
N \approx \frac{2\left[\mathcal{Q}^{-1}\left(P_{F A}\right)-\mathcal{Q}^{-1}\left(1-P_{M D}\right)\right]^{2}}{\left[\theta \cdot N_{c} \cdot S N R-\left(\frac{\rho^{2}-1}{\rho}\right)\right]^{2}},
$$

Clearly (8) and (9) verify the assertion made in (7). Figure 2 plots the sample complexity of the radiometer and the matched filter. From the figure it is easy to see that the sample complexity curves go to infinity as the SNR decreases to the SNR wall. 


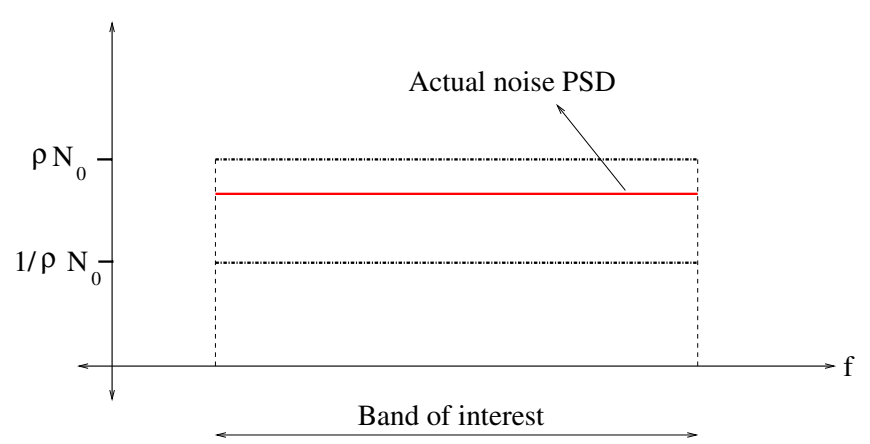

(a)

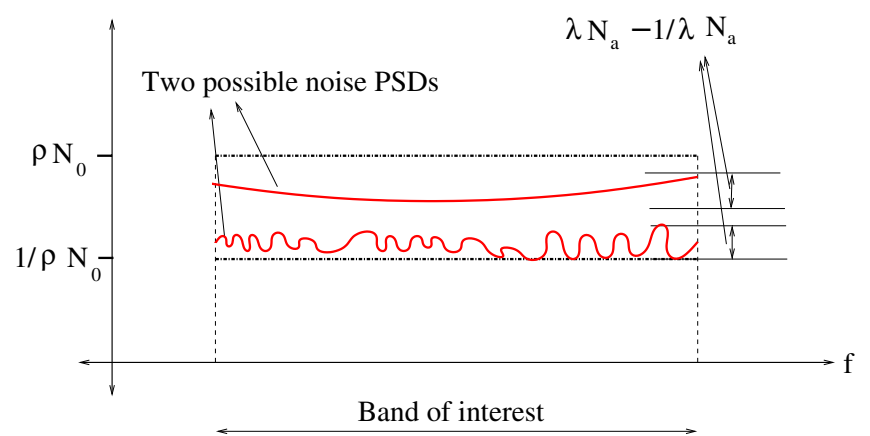

(b)

Fig. 1. Figure (a) describes the set of possible PSDs in $\mathbb{W}_{\rho}$ and Figure (b) describes the set of possible PSDs in $\widehat{\mathbb{W}}_{\rho, \lambda}$. The readers are advised to refer to [4] for a detailed description of this figure.

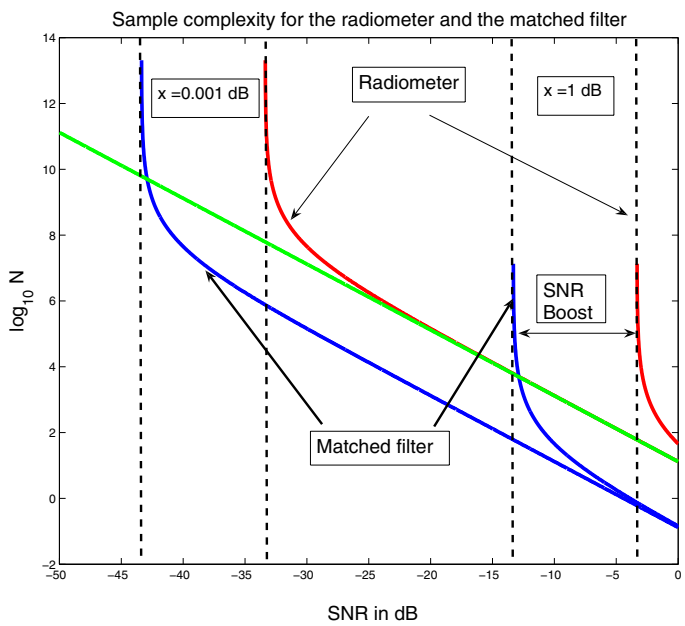

Fig. 2. This figure shows how the sample complexity ( $N$ as a function of SNR) for the radiometer and the matched filter varies as the SNR approaches the $S N R_{\text {wall }}$. (See Equations (8) and (9) and use $x=10 \log _{10} \rho, \theta=0.1, N_{c}=100, P_{F A}=0.1$, and $\left.P_{M D}=0.1\right)$. The two sets of curves for the matched filter and the radiometer correspond to $x=1 \mathrm{~dB}$ and $x=0.001 \mathrm{~dB}$ noise uncertainty. From the figure it is clear that the sample complexity approaches infinity as the SNR approaches the SNR wall.

\section{NOISE CALIBRATION AND DELAY COHERENCE}

In the previous section we reviewed the robustness limits of two simple detection schemes - energy and coherent detection. The SNR wall exists because of the uncertainty in the noise/interference process. The key signal-processing question is whether it is possible to reduce the uncertainty in the noise at run-time. The focus on run-time is important because any calibration done far in advance can get stale.

This leads to a tension. Ideally, we would like access to $\mathcal{H}_{0}$ in a parallel universe that is identical to our own except that the primary user is guaranteed to be absent. However, our detector is necessarily confined to our own universe where the presence or absence of the primary user is unknown. Any run-time calibration that aims to reduce the noise uncertainty must use data that might be corrupted by the presence of the primary signal.

We first review the idea of noise calibration in frequency domain for pilot detectors (see [4] for the details). Figure 3 describes the idea of noise calibration in frequency domain. Figure 3(a) shows the PSD of the received signal under hypothesis $\mathcal{H}_{1}$. The shaded region in the bottom is the PSD of the data carrying part of the signal, the solid region is the PSD of noise, and the delta function is the pilot tone. One possible approach to detect a narrowband pilot is the following: pass the received signal through an ideal band-pass filter centered around the pilot frequency, measure the power of the filtered signal and compare it to a threshold. The filtering operation reduces the noise power and hence boosts the SNR. As the fading process can spread the pilot tone in frequency there is a limit to the amount of coherent processing gain obtained by filtering. The residual noise power uncertainty in the filtered band leads to an SNR wall. One intuitive approach to reduce this uncertainty at run-time is to measure the noise power in another narrow band close to the pilot location and use it to calibrate the noise power in the band around the pilot tone (See Figure 3(b)). There will be some calibration error as the noise power in these two bands is not identical. However, this error can be much lower than the uncertainty in the noise power itself. Thus calibrating the noise power at run-time improves the SNR wall.

The key intuition is that run-time noise calibration is feasible only if the signal feature of interest, in this case the pilot tone, occupies a fraction of the total degrees of freedom. This allows for calibration via measurement from the unoccupied degrees of freedom. 


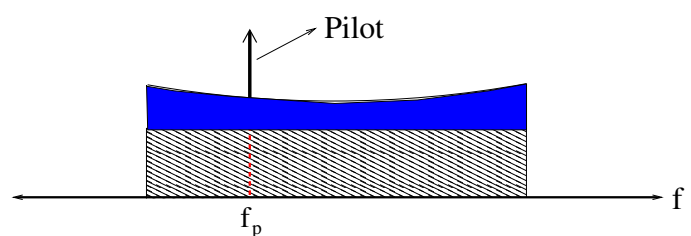

(a)

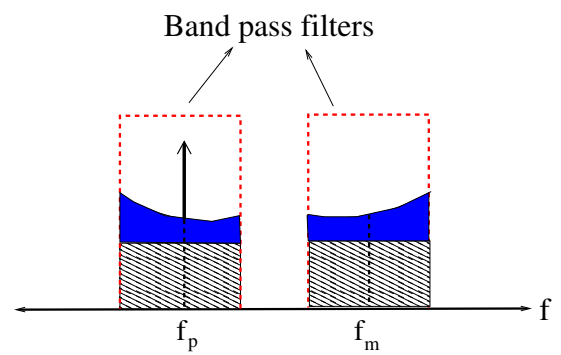

(b)

Fig. 3. This figure gives a pictorial description of noise calibration in frequency domain. Figure (a) shows the power spectrum of the received signal with colored noise. The shaded region corresponds to the signal power spectrum, the solid region corresponds to the noise power spectrum and the vertical arrow corresponds to the narrowband pilot tone. Figure (b) shows the technique used to perform noise calibration in frequency domain. The noise power (area of the solid region) in the band on the right is used to estimate the noise power in the band on the left. This technique is only feasible because the pilot occupies a small fraction of the total degrees of freedom.

\section{A. 50\%-duty-cycle PAM signal example}

The goal of this paper is to show that run-time noise calibration is a powerful technique and can be applied to improve robustness for a rich class of primary signals beyond the frequency domain example.

We now consider an example of a signal occupying a fraction of the underlying degrees of freedom. To be specific, consider signals that use only half of the available degrees of freedom in time rather than frequency. The signal $X[n]$ can be described as follows:

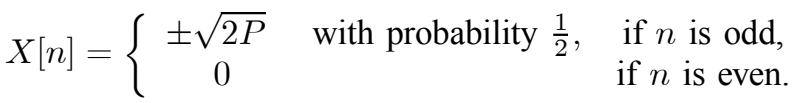

The average power of this signal is $P$ and this example is a caricature of practical signals whose amplitude is modulated using time-domain pulses. Such PAM signals come under the general category of cyclostationary signals [9]-[11]. Here we illustrate the basic idea of noise calibration for cyclostationary signals in the context of this simple caricature.

In this example, the average power measurements at 'even' time samples can be used to calibrate the noise in the 'odd' time samples.
1) No channel fading: Suppose that there was no fading. The natural test statistic is given by

$T(\mathbf{Y})=\left[\frac{2}{N} \sum_{n=1}^{\frac{N}{2}}|Y[2 n-1]|^{2}-\frac{2}{N} \sum_{n=1}^{\frac{N}{2}}|Y[2 n]|^{2}\right]$.

In this case it is easy to verify that $\mathbb{E}\left[T(\mathbf{Y}) \mid \mathcal{H}_{0}\right]=$ $\mathbb{E}\left[\frac{2}{N} \sum_{n=1}^{\frac{N}{2}}|W[2 n-1]|^{2}\right]-\mathbb{E}\left[\frac{2}{N} \sum_{n=1}^{\frac{N}{2}}|W[2 n]|^{2}\right]=0$ and $\mathbb{E}\left[T(\mathbf{Y}) \mid \mathcal{H}_{1}\right]=2 P \neq 0$. Therefore, it is clear that we can obtain infinite gains from noise calibration and hence there is no SNR wall if the noise is guaranteed to be wide sense stationary. The noise contribution to each expectation is identical by stationarity.

2) Flat fading: Assume that the channel fading is flat (i.e $H(\cdot)$ is just multiplication by a bandlimited scalar signal). For pilot-detection, this is enough to cause an SNR wall since it spreads the pilot tone over a finite bandwidth and thereby stops infinite coherent processing gains [4]. In this case however, the scalar multiplication can do nothing to the zero samples of the signal! Even time-samples continue to provide a clean view of the noise. Therefore, by the arguments above noise calibration becomes perfect. Noise color is not enough to stop this since the stationarity of the noise guarantees that the calibration term under hypothesis $\mathcal{H}_{0}$, $\frac{2}{N} \sum_{n=1}^{\frac{N}{2}}|Y[2 n]|^{2}=\frac{2}{N} \sum_{n=1}^{\frac{N}{2}}|W[2 n]|^{2}$ converges with probability one to $\frac{2}{N} \sum_{n=1}^{\frac{N}{2}}|W[2 n-1]|^{2}$ as $N$ gets large.

3) Frequency-selective fading: The previous result is somewhat surprising since the matched filter for pilot tones is not-robust to this kind of flat-fading uncertainty, even with noise calibration when the noise is colored. However, frequency-selective fading does introduce an SNR wall for this detector in (11). To illustrate the idea, consider the simplest possible frequency-selective fading: a pure delay. Let the received signal under hypothesis $\mathcal{H}_{1}$ be $Y[n]=X[n-l[n]]+W[n]$. In this case, we show that uncertainty in the delay $l[n]$ will lead to an SNR wall. To be concrete, consider a block-fading model in which the delay $l[n]$ is piecewise constant for $D_{c}$ time steps before taking on an independent realization. Since $D_{c}$ models the minimum time over which the delay of the fading process changes significantly, we call it the delay-coherence time. More generally, the delaycoherence time refers to the minimum time over which the shape of the impulse response of the fading process changes significantly.

\section{B. Robustness analysis}

For this simplest example of a fading process with finite $D_{c}$, the strategy of (11) is doomed to failure if 
the sums are taken beyond one coherence time. The signal will eventually corrupt both the even and odd time samples due to the random delay. By analogy with the case of matched filtering, a natural detection strategy is to compute the square of the test statistic within each coherence time and average that over multiple coherence times. The modified test statistic is given in (12).

Theorem 1: Let the received signal under hypothesis $\mathcal{H}_{1}$ in (1) be given by $Y[n]=X[n-l[n]]+W[n]$, with $X[n]$ given in (10), and $W[n]$ stationary uncertain noise independent of the signal. Assume that the distribution of the noise arises from the colored noise uncertainty set $\widehat{\mathbb{W}}_{\rho, \lambda}$. Suppose the delay fading satisfies:

- $l[n]$ is constant within a delay-coherence block and iid across different delay-coherence blocks.

- $l[n]$ is uniformly distributed on $\{0,1\}$.

Define, $S N R=\frac{P}{\sigma_{n}^{2}}$. Then, the detector with test-statistic given by

$$
\begin{aligned}
& T\left(\mathbf{Y}, D_{c}\right) \\
& =\frac{1}{M} \sum_{k=0}^{M-1}\left[\mid \sqrt{\frac{2}{D_{c}}} \sum_{n=1}^{D_{c} / 2}\left\{|Y[k M+2 n-1]|^{2}\right.\right. \\
& \left.\left.-\sqrt{\frac{2}{D_{c}}} \sum_{n=1}^{D_{c} / 2}|Y[k M+2 n]|^{2}\right\}\left.\right|^{2}\right],
\end{aligned}
$$

where $M=\frac{N}{D_{c}}$ is non-robust if

$$
S N R \leq \frac{-2\left(\frac{\lambda}{\rho}\right)+\sqrt{4\left(\frac{\lambda^{2}}{\rho^{2}}\right)+2 D_{c}\left(\frac{\rho^{2}}{\lambda^{2}}-\frac{\lambda^{2}}{\rho^{2}}\right)}}{D_{c}} .
$$

Proof: From (12) it is clear that

$$
\begin{aligned}
& \mathbb{E}\left[T\left(\mathbf{Y}, D_{c}\right) \mid \mathcal{H}_{i}\right]= \\
& \mathbb{E}\left[\left|\sqrt{\frac{2}{D_{c}}} \sum_{n=1}^{D_{c} / 2}\left\{|Y[2 n-1]|^{2}-|Y[2 n]|^{2}\right\}\right|^{2} \mid \mathcal{H}_{i}\right],
\end{aligned}
$$

for $i=0,1$. Since we are computing the square of the test statistic within each coherence block, assume without loss of generality that the delay in the first coherence block is zero, i.e., $l[n]=0$ for $n=1,2, \cdots, D_{c}$. The detector in (12) is non-robust if there exists a class of noise distributions such that the set of means under both hypotheses overlap. We now exhibit a specific class of noise distributions that satisfy this condition.

Assume $W[n]$ are iid random variables with marginals given by $W[n] \sim \mathcal{N}\left(0, \sigma^{2}\right)$ for some $\frac{\lambda}{\rho} \leq \sigma^{2} \leq \frac{\rho}{\lambda}$. The following lemma gives the desired quantities.

\section{Lemma 1:}

$$
\begin{aligned}
\mathbb{E}\left[T\left(\mathbf{Y}, D_{c}\right) \mid \mathcal{H}_{0}\right] & =4 \sigma^{4}, \\
\mathbb{E}\left[T(\mathbf{Y}, N) \mid \mathcal{H}_{1}\right] & =2 P^{2} D_{c}+8 P \sigma^{2}+4 \sigma^{4},
\end{aligned}
$$

where $\sigma^{2}=\mathbb{E}\left[|W[n]|^{2}\right]$.

Proof: See Appendix I.

Using Lemma 1, it is clear that the detector in (12) is non-robust if the set of means under both hypotheses overlap. This condition is equivalent to

$$
\begin{aligned}
& \min _{\frac{\lambda}{\rho} \sigma_{n}^{2} \leq \sigma^{2} \leq \frac{\rho}{\lambda} \sigma_{n}^{2}} 2 P^{2} D_{c}+8 P \sigma^{2}+4 \sigma^{4} \\
\leq & \max _{\frac{\lambda}{\rho} \sigma_{n}^{2} \leq \sigma^{2} \leq \frac{\rho}{\lambda} \sigma_{n}^{2}} 4 \sigma^{4} \\
\Rightarrow & P^{2} D_{c}+4\left(\frac{\lambda}{\rho}\right) P \sigma_{n}^{2}-2\left(\frac{\rho^{2}}{\lambda^{2}}-\frac{\lambda^{2}}{\rho^{2}}\right) \sigma_{n}^{4} \leq 0
\end{aligned}
$$

The last inequality in $S N R:=\frac{P}{\sigma_{n}^{2}}$ can be solved to give the required result by the quadratic formula.

This theorem suggests that it is the uncertainty corresponding to the frequency selectivity of the fading that induces limits on the detection of very weak signals that do not fully utilize all the degrees of freedom in timedomain. There are two interesting aspects of this result:

- The dependence of the SNR wall for this detector on the delay coherence time is $O\left(\frac{1}{\sqrt{D_{c}}}\right)$. This gain from increasing delay-coherence time is slower than the $O\left(\frac{1}{N_{c}}\right)$ gain for the matched filter, but it is better than the lack of any gain with coherence time for a primary signal that only uses half the degrees of freedom in the frequency domain [4].

- The SNR wall result so far does not depend on the color of the noise! The proposed detector in (12), while being intuitively very natural, is non-robust in the presence of frequency-selective fading, even when the noise is truly white (use $\lambda=1$ in (13)). As the next section shows, this is an artifact of the detection strategy used.

\section{Noise calibration in time domain}

In matched filtering, we obtained the approximate level of noise from noise power measurements in an adjacent band. In the current example, the test statistic is a difference and so intuitively, the sum can provide access to the approximate level of noise. Explicitly, compute the empirical power in the received signal $\widehat{P}=$ $\frac{1}{N} \sum_{n=1}^{N}|Y[n]|^{2}$. This converges to $P+\sigma^{2}$ under $\mathcal{H}_{1}$ and to $\sigma^{2}$ under $\mathcal{H}_{0}$ as $N \rightarrow \infty$, where $\sigma^{2}=\mathbb{E}\left[|W[n]|^{2}\right]$. We normalize the received signal with this empirical power estimate and compute the new test statistic given in (16). Intuitively, one can think of this as passing 
the received signal through an automatic gain controller (AGC), which is tuned such that its output is normalized to unit power.

Theorem 2: Let the received signal under hypothesis $\mathcal{H}_{1}$ in (1) be given by $Y[n]=X[n-l[n]]+W[n]$, with $X[n]$ given in (10), and $W[n]$ being independent of the signal with an unknown distribution from the colored noise uncertainty set $\widehat{\mathcal{W}}_{\rho, \lambda}$. Suppose the delay fading $l[n]$ satisfies:

- $l[n]$ is constant within a delay-coherence block and iid across different delay-coherence blocks.

- The length of the delay-coherence block is $D_{c}>2$.

- $l[n]$ is uniformly distributed on $\{0,1\}$.

Then, the detector with test-statistic given by

$$
\begin{aligned}
& \widetilde{T}\left(\mathbf{Y}, D_{c}\right) \\
& =\frac{1}{M} \sum_{k=0}^{M-1}\left[\mid \sqrt{\frac{2}{D_{c}}} \sum_{n=1}^{D_{c} / 2}\left\{|\widehat{Y}[k M+2 n-1]|^{2}\right.\right. \\
& \left.\left.-\sqrt{\frac{2}{D_{c}}} \sum_{n=1}^{D_{c} / 2}|\widehat{Y}[k M+2 n]|^{2}\right\}\left.\right|^{2}\right]
\end{aligned}
$$

where $M=\frac{N}{D_{c}}$ and $\widehat{Y}[n]=\frac{Y[n]}{\sqrt{\frac{1}{N} \sum_{n=1}^{N}|Y[n]|^{2}}}$ is nonrobust if

$$
S N R \leq\left(\frac{\rho}{\lambda}\right) \cdot \frac{2\left(1-\frac{1}{\sqrt{\lambda}}\right)}{\sqrt{D_{c}}} .
$$

Proof: Assume that a genie guarantees that the times when the delay $l[n]$ assumes an independent realization are integer multiples of $D_{c}$ itself. From (16) it is clear that

$$
\begin{aligned}
& \mathbb{E}\left[\widetilde{T}\left(\mathbf{Y}, D_{c}\right) \mid \mathcal{H}_{i}\right]= \\
& \mathbb{E}\left[\left|\sqrt{\frac{2}{D_{c}}} \sum_{n=1}^{D_{c} / 2}\left\{|\widehat{Y}[2 n-1]|^{2}-|\widehat{Y}[2 n]|^{2}\right\}\right|^{2} \mid \mathcal{H}_{i}\right],
\end{aligned}
$$

for $i=0,1$. Since we are computing the square of the test statistic within each coherence block, assume without loss of generality that the delay in the first coherence block is zero, i.e., $l[n]=0$ for $n=$ $1,2, \cdots, D_{c}$. The detector in (16) is non-robust if there exist two distinct noise distributions $\mathcal{W}_{1}, \mathcal{W}_{2} \in \widehat{\mathbb{W}}_{\rho, \lambda}$ such that $\mathbb{E}_{\mathcal{W}_{1}}\left[\widetilde{T}\left(\mathbf{Y}, D_{c}\right) \mid \mathcal{H}_{1}\right]=\mathbb{E}_{\mathcal{W}_{2}}\left[\widetilde{T}\left(\mathbf{Y}, D_{c}\right) \mid \mathcal{H}_{0}\right]$. We now exhibit a specific class of noise distributions that satisfy this condition.

Let $M[n]$ be an iid white Gaussian noise process, with the variance $\sigma_{m}^{2} \in\left[\frac{\lambda}{\rho} \sigma_{n}^{2}, \frac{\rho}{\lambda} \sigma_{n}^{2}\right]$. Let $W[n]=M[n]-$ $\alpha M[n-1]$ for some $\alpha$ within some small range $0 \leq$ $|\alpha| \leq \alpha_{\max }$.
To apply the central limit theorem, define

$$
\begin{aligned}
& \left(G_{1}, G_{2}\right):= \\
& \left(\sqrt{\frac{2}{D_{c}}} \sum_{n=1}^{D_{c} / 2}|\widehat{Y}[2 n-1]|^{2}, \sqrt{\frac{2}{D_{c}}} \sum_{n=1}^{D_{c} / 2}|\widehat{Y}[2 n]|^{2}\right) .
\end{aligned}
$$

The parameters of the jointly Gaussian approximation for $\left(G_{1}, G_{2}\right)$ are computed in the following lemma.

Lemma 2: Assume $N \gg 1$. Then, the statistics of $\left(G_{1}, G_{2}\right)$ under hypothesis $\mathcal{H}_{1}$ are given by the mean and covariance

$$
\begin{aligned}
\bar{m} & :=\left(\begin{array}{c}
\mathbb{E} G_{1} \\
\mathbb{E} G_{2}
\end{array}\right), \\
\Lambda & :=\left(\begin{array}{cc}
\operatorname{Var}\left(G_{1}\right) & \operatorname{Cov}\left(G_{1}, G_{2}\right) \\
\operatorname{Cov}\left(G_{1}, G_{2}\right) & \operatorname{Var}\left(G_{1}\right)
\end{array}\right),
\end{aligned}
$$

taking values

$$
\begin{aligned}
\bar{m} & =\frac{1}{\kappa}\left(\begin{array}{c}
\sqrt{\frac{D_{c}}{2}}\left(2 P+\sigma^{2}\right) \\
\sqrt{\frac{D_{c}}{2}} \sigma^{2}
\end{array}\right), \\
\Lambda & =\frac{1}{\kappa^{2}}\left(\begin{array}{cc}
8 P \sigma^{2}+2 \sigma^{4} & 4 \alpha^{2} \sigma_{m}^{4}\left[\frac{D_{c}-2}{D_{c}}\right] \\
4 \alpha^{2} \sigma_{m}^{4}\left[\frac{D_{c}-2}{D_{c}}\right] & 2 \sigma^{4}
\end{array}\right),
\end{aligned}
$$

where $\kappa:=\frac{1}{P+\sigma^{2}}, \sigma^{2}=\left(1+\alpha^{2}\right) \sigma_{m}^{2}$. The corresponding statistics under the hypothesis $\mathcal{H}_{0}$ are computed by substituting $P=0$ in (20).

Proof: See Appendix II.

From (18) we have

$$
\begin{aligned}
\mathbb{E}\left[\widetilde{T}\left(\mathbf{Y}, D_{c}\right) \mid \mathcal{H}_{1}\right]= & \mathbb{E}\left[\left|G_{1}-G_{2}\right|^{2}\right] \\
= & \operatorname{Var}\left(G_{1}\right)+\operatorname{Var}\left(G_{2}\right)- \\
& 2 \operatorname{Cov}\left(G_{1}, G_{2}\right)+\left(\mathbb{E} G_{1}-\mathbb{E} G_{2}\right)^{2} .
\end{aligned}
$$

Substituting (20) in (21) gives

$$
\begin{aligned}
\mathbb{E}\left[\widetilde{T}\left(\mathbf{Y}, D_{c}\right) \mid \mathcal{H}_{1}\right]= & \frac{4\left[\left(1+\alpha^{2}\right) 2 P+\left(1+\alpha^{4}\right) \sigma_{m}^{2}\right] \sigma_{m}^{2}}{\left(P+\left(1+\alpha^{2}\right) \sigma_{m}^{2}\right)^{2}} \\
& +\frac{8 \alpha^{2} \sigma_{m}^{4}\left(\frac{2}{D_{c}}\right)+\left(\frac{D_{c}}{2}\right) 4 P^{2}}{\left(P+\left(1+\alpha^{2}\right) \sigma_{m}^{2}\right)^{2}}
\end{aligned}
$$

Setting $P=0$ in (22) gives

$$
\begin{aligned}
\mathbb{E}\left[\widetilde{T}\left(\mathbf{Y}, D_{c}\right) \mid \mathcal{H}_{0}\right] & =\frac{4\left(1+\alpha^{4}\right)+8\left(\frac{2}{D_{c}}\right) \alpha^{2}}{\left(1+\alpha^{2}\right)^{2}} \\
& =4\left[1-\frac{2 \alpha^{2}\left(1-\frac{2}{D_{c}}\right)}{\left(1+\alpha^{2}\right)^{2}}\right] \\
& \leq 4,
\end{aligned}
$$


$\forall D_{c}>2$. From (22) and (23), it is clear that the means of the test statistic under both hypotheses overlap if

$$
\begin{aligned}
& 4 \geq \frac{4\left[\left(1+\alpha_{\max }^{2}\right) 2 P+\left(1+\alpha_{\max }^{4}\right) \sigma_{m}^{2}\right] \sigma_{m}^{2}}{\left(P+\left(1+\alpha_{\max }^{2}\right) \sigma_{m}^{2}\right)^{2}} \\
&+\frac{8 \alpha_{\max }^{2} \sigma_{m}^{4}\left(\frac{2}{D_{c}}\right)+\left(\frac{D_{c}}{2}\right) 4 P^{2}}{\left(P+\left(1+\alpha_{\max }^{2}\right) \sigma_{m}^{2}\right)^{2}} \\
& \Rightarrow 0 \stackrel{(a)}{\geq}-\left(1-\frac{2}{D_{c}}\right) 8 \alpha_{\max }^{2} \sigma_{m}^{4}+\left(\frac{D_{c}}{2}-1\right) 4 P^{2} \\
& \Rightarrow S N R \leq \frac{2 \alpha_{\max }}{\sqrt{D_{c}}}\left(\frac{\sigma_{m}^{2}}{\sigma_{n}^{2}}\right) \leq\left(\frac{\rho}{\lambda}\right) \frac{2 \alpha_{\max }}{\sqrt{D_{c}}} .
\end{aligned}
$$

In the above chain of inequalities, $(a)$ follows by completing squares on the right hand side.

To prove the result of the theorem we need to give conditions on $\alpha_{\max }$ such that the distribution of the noise process $W[n]$ lies in $\widehat{\mathcal{W}}_{\rho, \lambda}$.

Lemma 3: Let $\alpha_{\max }=1-\frac{1}{\sqrt{\lambda}}$, where $\lambda>1$ is the parameter in the definition of the colored noise uncertainty set $\widehat{\mathcal{W}}_{\rho, \lambda}$. Then the class of noise processes defined by $W[n]=M[n]-\alpha M[n-1]$ (for white $M[n]$ with variance $\left.\sigma_{m}^{2} \in\left[\frac{\lambda}{\rho} \sigma_{n}^{2}, \frac{\rho}{\lambda} \sigma_{n}^{2}\right]\right)$ have distributions $W \in \widehat{\mathcal{W}}_{\rho, \lambda}$ for all $0 \leq|\alpha| \leq \alpha_{\max }$.

Proof: See Appendix III.

By Lemma 3, setting $\alpha_{\max }=1-\frac{1}{\sqrt{\lambda}}$ in (24) gives us the required result.

\section{INTERPRETING THE “DELAY-COHERENCE TIME"}

In real-world wireless environments, the channel response tends to change continuously due to relative motion in space-time. Coherence time is a useful shorthand for the time required for the channel to change "significantly." What constitutes a significant change is a matter of engineering judgement and depends on the context.

The magnitude-coherence time is the minimum time over which the magnitude response of the fading process changes significantly. This coherence-time tends to be inversely proportional to the Doppler spread of the channel. Traditionally, a significant change in the magnituderesponse leads to a change in the "water-filling" power allocation across sub-channels. By contrast, the phasecoherence time is the minimum time over which the phase of an individual channel tap changes significantly. This is relevant in the context of how long a matchedfilter can operate as well as for beamforming in MIMO contexts. On the other hand, the delay-coherence time is the minimum time over which the shape of the impulse response changes significantly. Alternatively, we can also think of it as the minimum time over which the relative phase-response changes significantly.
In most traditional communication systems, receivers have a phase locked loop (PLL). However, for sensors trying to detect the presence/absence of primary signals it is not possible to have a phase lock since the receiver is not even sure whether the primary signal is present or absent. This leads to a frequency offset error that in time leads to missed or extra samples. This possibility introduces a finite delay-coherence time even when the sensor is not physically in motion. It also shows how a change in the delay is not only the simplest possible example, it is also unavoidable in real-world systems.

To further understand the nature of the delaycoherence time $D_{c}$, it is useful to consider two cases:

- Assume that the delay spread of the channel is significantly larger than 1 sample and all of the channel taps arise from the sum of many paths. (There is no dominant line-of-sight path) As these path-lengths change over time, all of the taps will get different values. In such a case, the delay-coherence time $D_{c}$ and the traditional phase-coherence time $N_{c}$ (or equivalently, the reciprocal of the bandwidth of the fading process) are the same since the relative phase response can change significantly in this time. This is the model that is likely to hold for wideband primary users being detected in urban environments at significant distances from their transmitters. This basically corresponds to when ISI is significant.

- Now suppose there is only one dominant line-ofsight path that is arriving at the spectrum sensor, but is subject to local reflections in the near neighborhood of the sensor. (e.g. A user in an airplane) The delay spread of the channel can be smaller than 1 sample. In this case, there will just be a single tap in the filter. The amplitude might change rapidly since the local paths can go in and out of phase with each other, but the overall delay is not going to be changing as fast. In such cases, the delaycoherence time $D_{c}>N_{c}$ and the dominant effect may very well be the clock skew of the sensor's local oscillator relative to the primary oscillator. In such cases, the factor difference between $D_{c}$ and $N_{c}$ is comparable to the factor difference between the signal bandwidth and the carrier frequency.

\section{DISCUSSION AND CONCLUSIONS}

\section{A. Comparing different SNR walls}

In this paper we have shown the existence and computed the location of SNR walls for the $50 \%$-duty-cycle example with noise calibration. We now compare the 




Fig. 4. The location of SNR walls for various detection algorithms is plotted as a function of the delay-coherence time. The curve with 'triangle' markers (blue) is the location of the SNR wall for the energy detector. The dashed curve (red) is the location of the SNR wall for the matched filter assuming $50 \%$ of the power is in the pilot. The dashed-dotted curve (black) is the location of the SNR wall for the $50 \%$-duty-cycle example without noise calibration. Finally, the solid curve (green) is the location of the SNR wall for the $50 \%$-duty-cycle example with noise calibration. These curves were obtained from Equations (2), (3), (13), and (17) respectively.

robustness for this detector to the robustness of other detection algorithms. Figure 4 plots the SNR wall locations for the radiometer, the matched filter, and the $50 \%$ duty-cycle example with and without noise calibration as a function of the channel-coherence time $N_{c}$. In this example, the delay-coherence time is assumed to be the same as the phase-coherence time, i.e., $N_{c}=D_{c}$. In this plot we have assumed $\theta=0.5$ (See (3)) for the matched filter and the noise uncertainty parameters are $\rho=10$ and $\lambda=10^{\frac{1}{10}}$.

In Figure 4, the curve with 'triangle' markers corresponds to the energy detection SNR wall, and this is independent of the channel-coherence time. The dashed curve corresponds to the matched filter. The dasheddotted curved corresponds to the $50 \%$-duty-cycle example without noise calibration (See (13)), and the solid curve represents the SNR wall for the $50 \%$-duty-cycle example with noise calibration (See (17)).

Notice that in Figure 4, the matched filter SNR wall is better than the SNR wall for the $50 \%$-duty-cycle example with noise calibration for $N_{c} \geq 300$. The $50 \%$-dutycycle signaling scheme has a significant loss in primary data rate as compared to an optimal capacity achieving signaling scheme since it is effectively throwing away more than half the degrees of freedom. In general as the signaling scheme makes better use of the degrees of freedom the robustness gains will diminish.

\section{B. Concluding remarks}

In this paper we consider the example of a $50 \%$-dutycycle PAM signal to demonstrate the robustness gains achievable by run-time noise calibration. This example suggests that the robustness gains for feature detection are coming implicitly from run-time calibration of the noise model based on the observations themselves.

The noise-calibration gains presented in this paper are not specific to the $50 \%$ duty-cycle example. Our analysis can be applied to any PAM signal with an arbitrary dutycycle. Furthermore, these techniques can also be applied to cyclostationary feature detectors [16]. Knowledge of deterministic components of the primary signal such as pilot tones give rise to SNR walls that scale as $\frac{1}{N_{c}}$ with phase-coherence time $N_{c}$ whereas knowledge of purely structural features of the data signaling strategy give rise to SNR walls that scale only as $\frac{1}{\sqrt{D_{c}}}$ with delaycoherence time $D_{c}$. This suggests that unless the delaycoherence time is significantly larger than the phasecoherence time, it is unlikely that feature-detection will be an attractive choice to increase the robustness of detecting very weak primary users.

\section{APPENDIX I}

PROOF OF LEMMA 1

Under hypothesis $\mathcal{H}_{0}: Y[n]=W[n] \sim \mathcal{N}\left(0, \sigma^{2}\right)$ for all $n>0$. Using this in (14), we get

$$
\begin{aligned}
& \mathbb{E}\left[T\left(\mathbf{Y}, D_{c}\right) \mid \mathcal{H}_{0}\right]= \\
& \mathbb{E}\left[\left|\sqrt{\frac{2}{D_{c}}} \sum_{n=1}^{D_{c} / 2}\left\{|W[2 n-1]|^{2}-|W[2 n]|^{2}\right\}\right|^{2}\right]
\end{aligned}
$$

$|W[2 n-1]|^{2}-|W[2 n]|^{2}$ are iid random variables with zero mean and variance $4 \sigma^{4}$. Using the Central Limit Theorem [17], we get

$\sqrt{\frac{2}{D_{c}}} \sum_{n=1}^{D_{c} / 2}\left\{|W[2 n-1]|^{2}-|W[2 n]|^{2}\right\} \sim \mathcal{N}\left(0,4 \sigma^{4}\right)$.

Using this approximation in (25), we get $\mathbb{E}\left[T\left(\mathbf{Y}, D_{c}\right) \mid \mathcal{H}_{0}\right]=4 \sigma^{4}$.

Under hypothesis $\mathcal{H}_{1}$ : Without loss of generality we can assume $l[n]=0$. So, we have $Y[2 n-1]=X[2 n-$ $1]+W[2 n-1]$ and $Y[2 n]=X[2 n]+W[2 n]=W[2 n]$. This implies that $|Y[2 n-1]|^{2}-|Y[2 n]|^{2}$ are iid random variables with mean $2 P$ and variance $8 P \sigma^{2}+4 P^{2}$. Again 
using the Central Limit Theorem [17], we get

$$
\begin{aligned}
& \sqrt{\frac{2}{D_{c}}} \sum_{n=1}^{D_{c} / 2}\left\{|Y[2 n-1]|^{2}-|Y[2 n]|^{2}\right\} \sim \\
& \mathcal{N}\left(\sqrt{\frac{D_{c}}{2}}(2 P), 8 P \sigma^{2}+4 \sigma^{4}\right) .
\end{aligned}
$$

Using this approximation we get

$$
\begin{aligned}
\mathbb{E}\left[T\left(\mathbf{Y}, D_{c}\right) \mid \mathcal{H}_{1}\right] & =8 P \sigma^{2}+4 \sigma^{4}+\left(\sqrt{\frac{D_{c}}{2}}(2 P)\right)^{2} \\
& =8 P \sigma^{2}+4 \sigma^{4}+2 P^{2} D_{c} .
\end{aligned}
$$

\section{APPENDIX II}

PROOF OF LEMMA 2

Recall $\widehat{Y}[n]=\frac{Y[n]}{\sqrt{\frac{1}{N} \sum_{n=1}^{N}|Y[n]|^{2}}}$. Since we are interested in robustness results, we assume that $N$ is very large. Specifically, we assume that $\frac{1}{N} \sum_{n=1}^{N} Y^{2}[n]=$ $P+\sigma^{2}$ under hypothesis $\mathcal{H}_{1}$ and $\frac{1}{N} \sum_{n=1}^{N} Y^{2}[n]=\sigma^{2}$ under hypothesis $\mathcal{H}_{0}$, where $\sigma^{2}=\left(1+\alpha^{2}\right) \sigma_{m}^{2}$.

Under hypothesis $\mathcal{H}_{1}$ : From (19), we can write $G_{1}=$ $K_{1} S_{1}$, and $G_{2}=K_{1} S_{2}$, where

$$
S_{1}=\sum_{n=1}^{\frac{D_{c}}{2}} Y^{2}[2 n-1], \quad S_{2}=\sum_{n=1}^{\frac{D_{c}}{2}} Y^{2}[2 n]
$$

and $K_{1}=\sqrt{\frac{2}{D_{c}}} \cdot \frac{1}{P+\sigma^{2}}$. Now, $\mathbb{E} S_{1}=\sum_{n=1}^{\frac{D_{c}}{2}} \mathbb{E} Y^{2}[2 n-$ $1]=\sum_{n=1}^{\frac{D_{c}}{2}} \mathbb{E}(X[2 n-1]+W[2 n-1])^{2}=$ $\frac{D_{c}}{2}\left[2 P+\sigma^{2}\right]$. Similarly, $\mathbb{E} S_{2}=\sum_{n=1}^{\frac{D_{c}}{2}} \mathbb{E} Y^{2}[2 n]=$ $\sum_{n=1}^{\frac{D_{c}}{2}} \mathbb{E}(W[2 n])^{2}=\frac{D_{c}}{2}\left[\sigma^{2}\right]$. Here we have used the fact that the random delay in the current coherent block is $l[n]=0$. Therefore, $X[2 n-1]$ is $\pm \sqrt{2 P}$ with probability $\frac{1}{2}$ for each sign and $X[2 n]=0$. Hence, we get $\mathbb{E}\left[G_{1}\right]=K_{1} \mathbb{E}\left[S_{1}\right]=\frac{1}{P+\sigma^{2}}\left(\frac{D_{c}}{2}\right)\left[2 P+\sigma^{2}\right]$ and $\mathbb{E}\left[G_{2}\right]=K_{1} \mathbb{E}\left[S_{2}\right]=\frac{1}{P+\sigma^{2}} \frac{D_{c}}{2}\left[\sigma^{2}\right]$.

We now compute the second-order statistics of $\left(G_{1}, G_{2}\right)$. By definition, we have $\mathbb{E}\left(G_{1}-\mathbb{E} G_{1}\right)^{2}=$ $K_{1}^{2} \mathbb{E}\left(S_{1}-\mathbb{E} S_{1}\right)^{2}$ and $\mathbb{E}\left(G_{2}-\mathbb{E} G_{2}\right)^{2}=K_{1}^{2} \mathbb{E}\left(S_{2}-\mathbb{E} S_{2}\right)^{2}$.
Now,

$$
\begin{aligned}
& \mathbb{E} S_{1}^{2} \\
= & \mathbb{E}\left[\sum_{n=1}^{\frac{D_{c}}{2}} Y^{2}[2 n-1]\right]^{2} \\
= & \sum_{n=1}^{\frac{D_{c}}{2}} \mathbb{E}\left(Y^{4}[2 n-1]\right) \\
& +\sum_{m \neq n} \mathbb{E}\left(Y^{2}[2 n-1] Y^{2}[2 m-1]\right) .
\end{aligned}
$$

Using the fact that $Y[2 n-1]=X[2 n-1]+W[2 n-1]$, we can easily show that $\mathbb{E}\left(Y^{4}[2 n-1]\right)=4 P^{2}+12 P \sigma^{2}+3 \sigma^{4}$ and $\mathbb{E}\left(Y^{2}[2 n-1] Y^{2}[2 m-1]\right)=4 P^{2}+4 P \sigma^{2}+\sigma^{4}$. Substituting this in (26) we get

$$
\begin{aligned}
\mathbb{E} S_{1}^{2}= & \frac{D_{c}}{2}\left[4 P^{2}+12 P \sigma^{2}+3 \sigma^{4}\right] \\
& +\frac{D_{c}}{2}\left(\frac{D_{c}}{2}-1\right)\left[4 P^{2}+4 P \sigma^{2}+\sigma^{4}\right]
\end{aligned}
$$

Therefore,

$$
\begin{aligned}
& \operatorname{Var}\left(S_{1}\right)=\mathbb{E} S_{1}^{2}-\left(\mathbb{E} S_{1}\right)^{2}=\frac{D_{c}}{2}\left[8 P \sigma^{2}+2 \sigma^{4}\right] \\
& \Rightarrow \operatorname{Var}\left(G_{1}\right)=K_{1}^{2} \operatorname{Var}\left(S_{1}\right)=\frac{\left[8 P \sigma^{2}+2 \sigma^{4}\right]}{\left(P+\sigma^{2}\right)^{2}}
\end{aligned}
$$

Identical computations show that $\operatorname{Var}\left(G_{2}\right)=\frac{\left[2 \sigma^{4}\right]}{\left(P+\sigma^{2}\right)^{2}}$.

$$
\begin{aligned}
& \mathbb{E}\left[S_{1} S_{2}\right]= \\
& \mathbb{E}\left[\left(\sum_{n=1}^{\frac{D_{c}}{2}}(X[2 n-1]+M[2 n-1]-\alpha M[2 n-2])^{2}\right)\right. \\
& \left.\left(\sum_{m=1}^{\frac{D_{c}}{2}}(M[2 m]-M[2 m-1])^{2}\right)\right]
\end{aligned}
$$

Expanding this product and taking expectations we get a total of $\left(\frac{D_{c}}{2}\right)^{2}$ terms. The terms can be grouped into two groups of the form $\mathbb{E}\left[(X[3]+M[3]-\alpha M[2])^{2}(M[2]-M[1])^{2}\right], \quad$ and $\mathbb{E}\left[(X[3]+M[3]-\alpha M[2])^{2}(M[6]-M[5])^{2}\right]$. The first group has exactly one $M[\cdot]$ term in common, while the second group has no $M[\cdot]$ terms in common. By standard computations, we can see that the expectation of each term in group one is $(2 P) \sigma^{2}+\sigma^{4}+2 \alpha^{2} \sigma_{m}^{4}$ and the expectation of each term in group two is $(2 P) \sigma^{2}+\sigma^{4}$. The number of terms in group one is $\left(D_{c}-2\right)$ and the number of terms in group two is 


$$
\begin{aligned}
& \left(\frac{D_{c}}{2}\right)^{2}-\left(D_{c}-2\right) \text {. Using this in }(27) \text {, we get } \\
& \mathbb{E}\left[S_{1} S_{2}\right]=\left(D_{c}-2\right)\left[(2 P) \sigma^{2}+\sigma^{4}+2 \alpha^{2} \sigma_{m}^{4}\right] \\
& +\left[\left(\frac{D_{c}}{2}\right)^{2}-\left(D_{c}-2\right)\right]\left[(2 P) \sigma^{2}+\sigma^{4}\right] \\
& \Rightarrow \operatorname{Cov}\left(G_{1}, G_{2}\right)=K_{1}^{2} \operatorname{Cov}\left(S_{1}, S_{2}\right)=\left[\frac{4 \alpha^{2} \sigma_{m}^{4}}{\left(P+\sigma^{2}\right)^{2}}\right]\left[\frac{D_{c}-2}{D_{c}}\right] .
\end{aligned}
$$

\section{APPENDIX III}

PROOF OF LEMMA 3

Let $W[n]=M[n]-\alpha M[n-1]$, where $M[n]$ is an iid Gaussian noise process with variance $\sigma_{m}^{2} \in\left(\frac{\lambda}{\rho} \sigma_{n}^{2}, \frac{\rho}{\lambda} \sigma_{n}^{2}\right)$. The noise process $\{W[n]\}$ certainly has a distribution in $\widehat{\mathcal{W}}_{\rho, \lambda}$ if

$$
\frac{1}{\lambda} \leq|H(f)|^{2} \leq \lambda, \quad \forall f \in\left[-\frac{1}{2}, \frac{1}{2}\right] .
$$

Here $H(f)=1-\alpha e^{j 2 \pi f}$, is the DTFT of the discrete time filter with impulse response $h[0]=1, h[1]=-\alpha$. Thus, we have $|H(f)|^{2}=1-2 \alpha \cos (2 \pi f)+\alpha^{2}$. Clearly, we have $(1-|\alpha|)^{2} \leq|H(f)|^{2} \leq(1+|\alpha|)^{2}$. Using this in (28), we conclude that $\{W[n]\}$ has a distribution in $\widehat{\mathcal{W}}_{\rho, \lambda}$ if

$$
\begin{array}{r}
\frac{1}{\lambda} \leq(1-|\alpha|)^{2} \leq(1+|\alpha|)^{2} \leq \lambda \\
\Leftrightarrow|\alpha| \leq 1-\frac{1}{\sqrt{\lambda}}=: \alpha_{\max } .
\end{array}
$$

\section{ACKNOWLEDGEMENTS}

This work was supported in part by Sumitomo Electric and NSF grants ANI-0326503, CCF-0635372, and CNS-0627161. The authors also thank the anonymous reviewers as well as Mubaraq Mishra and Kristen Ann Woyach for their feedback.

\section{REFERENCES}

[1] R. Tandra, S. M. Mishra, and A. Sahai, "What is a spectrum hole and what does it take to recognize one?" Proc. IEEE, submitted for publication.

[2] A. Sahai, N. Hoven, and R. Tandra, "Some fundamental limits on cognitive radio," in Forty-second Allerton Conference on Communication, Control, and Computing, Monticello, IL, Oct. 2004.

[3] S. Shellhammer, "Numerical spectrum sensing requirements," in IEEE 802.22-06/0088r0, June 2006.

[4] R. Tandra and A. Sahai, "SNR walls for signal detection," IEEE Journal on Selected Topics in Signal Processing, vol. 2, pp. 4 - 17, Feb. 2008.

[5] S. Shellhammer and R. Tandra, "Performance of the power detector with noise uncertainty," in IEEE 802.2206/0134r0, July 2006. [Online]. Available: "http://ieee802. org/22/Meeting_documents/2006_July/22-06-0134-00-0000_ Performance-of-the-power-detector-with-Noise-Uncertainty. ppt"
[6] - "An evaluation of DTV pilot power detection," in IEEE 802.22-06/0188r0, September 2006. [Online]. Available: "http://ieee802.org/22/Meeting_documents/2006_Sept/ 22-06-0188-00-0000-An-Evaluation-of-DTV-Pilot-Power-Detection. ppt"

[7] D. Cabric, A. Tkachenko, and R. W. Brodersen, "Experimental study of spectrum sensing based on energy detection and network cooperation," in the proceedings of IEEE MILCOM 2006.

[8] A. Sonnenschein and P. M. Fishman, "Radiometric detection of spread-spectrum signals in noise of uncertain power," IEEE Trans. Aerosp. Electron. Syst., vol. 28, pp. 654-660, July 1992.

[9] W. A. Gardner, "The spectral correlation theory of cyclostationary time-series," Signal Processing, vol. 11, pp. 13-36, July 1986.

[10] - Statistical Spectral Analysis: A Nonprobabilistic Theory. Englewood Cliffs, NJ: Prentice-Hall, 1987.

[11] - Introduction to Random Processes with Applications to Signals and Systems. New York: Macmillan, 1985.

[12] - "Signal interception: A unifying theoretical framework for feature detection," IEEE Trans. Commun., vol. 36, pp. 897906, Aug. 1988.

[13] $\longrightarrow$, "Signal interception: Performance advantages of cyclicfeature detectors," IEEE Trans. Commun., vol. 40, pp. 149-159, Jan. 1992.

[14] "Spectrum policy task force report," Federal Communications Commision, Tech. Rep. 02-135, Nov 2002. [Online]. Available: http://hraunfoss.fcc.gov/edocs $\$ \backslash \$$ public/ attachmatch/DOC-228542A1.pdf

[15] R. Tandra and A. Sahai, "SNR walls for feature detectors," in Proc. of 2nd IEEE International Symposium on New Frontiers in Dynamic Spectrum Access Networks, April 2007, pp. 559570.

[16] — , "SNR walls for cyclostationary feature detectors," In preparation for submission to IEEE Transactions on Signal Processing, 2008.

[17] R. Durrett, Probability: Theory and Examples, 3rd ed. Belmont: Duxbury Press, 2004. 